\title{
Mesenchymal stem cells home to but do not modulate acute renal injury in a canine model
}

\author{
Gabr H. ${ }^{1}$, Abdel Aziz W. Y. ${ }^{2}$, Zahran M. E. ${ }^{2}$, Autaifi M. A. ${ }^{2}$, Aboul-Hassan G. M. ${ }^{2}$, Al-Akabawy G. ${ }^{3}$, \\ Gomaa M. ${ }^{2}$
}

${ }^{1}$ Clinical Pathology Department, Faculty of Medicine, Cairo University, Cairo, Egypt

${ }^{2}$ Anatomy Department, Faculty of Medicine, Azhar University, Cairo, Egypt

${ }^{3}$ Anatomy Department, Faculty of Medicine, Menofia University, Shebin elkom, Egypt

\section{Email address:}

halagabr@kasralainy.edu.eg (Gabr H.)

\section{To cite this article:}

Gabr H., Abdel Aziz W. Y., Zahran M. E., Autaifi M. A., Aboul-Hassan G. M., Al-Akabawy G., Gomaa M.. Mesenchymal Stem Cells Home to but do not Modulate Acute Renal Injury in a Canine Model. International Journal of Biomedical Science and Engineering.

Vol. 2, No. 6, 2014, pp. 56-66. doi: 10.11648/j.ijbse.20140206.12

\begin{abstract}
Background: Acute kidney injury (ARI) is a common clinical event that occurs in $5-35 \%$ of all hospitalized patients and is associated with a two- to five fold increased mortality risk., with no significant improvement on pharmacologic therapy. The need for better treatment strategies for ARI called for cellular based strategies aiming to regenerate damaged tissues. Mesenchymal stem cells (MSCs) hold special promise in attenuating kidney injury, since nephrons are largely of mesenchymal origin. Objectives: The objective of this study is to evaluate the role of bone marrow derived (MSCs) in regeneration of post-ischemic acute renal injury in mongrel dogs. Material and Methods: Fifteen adult male mongrel dogs were used in this study. Animals were divided into: Group I, normal group (3 dogs); Group II, nine dogs subjected to ischemic/reperfusion injury (IRI) by clamping both renal pedicles for 60 minutes and not subjected to stem cell therapy which were further divided into: 6 dogs without sham injection, 3 sacrificed 3 days and 3 after 7 days after IRI and 3 dogs with sham injection sacrificed 7 days after IRI. and Group III ( 3 dogs) which underwent IRI and received systemic autologous MSC injection. This group underwent aspiration of 15-20 ml bone marrow from posterior iliac spine, mononuclear cell separation and MSC separation. MSCs were tagged by iron oxide and injected in a dose of 2 million $/ \mathrm{kg}$ systemically through the femoral vein. Evaluation of Therapeutic Effect: 1. Functional evaluation of renal functions using serum creatinine preoperative and 30 minutes, 1 day, 2days, 4days, 7days postoperative.2. Renal histology and injury scores: using H\&E, PAS using morphometry. 3. Tracing of injected MSCs (homing) in renal tissues using Prussian blue staining . 4. Immunohistochemical evaluation of the apoptosis and proliferative capacity of MSCs using caspase3 and KI 67. Results: Bone marrow derived MSCs were found in the renal cortex and medulla, epithelial lining of the cortical tubules, glomeruli, the Bowman's space, in some peritubular capillaries and among the epithelial lining of the renal tubules of treated animals. However,MSCs did not undergo transdifferentiation to renal cells, There was no difference in the regeneration, apoptosis or proliferation between treated and nontreated animals. Conclusions: Injected MSCs homed to but did not affect regeneration of renal tissue after acute injury. Although MSCs did not prove to differentiate into renal tissue, they can be used as vehicles for cytokines or growth factors as they home to injured sites.
\end{abstract}

Keywords: Stem Cell Therapy, Acute Renal Failure, Mesenchymal Stem Cells

\section{Introduction}

The kidney is a complex organ consisting of a number of cell populations functioning together to perform tightly controlled and complex processes. Acute kidney injury (ARI) is a common clinical event that occurs in $5-35 \%$ of all hospitalized patients and is associated with a two- to five fold increased mortality risk. (1), with no significant improvement on pharmacologic therapy (2).

The term ARI encompasses a spectrum of diseases ranging from minor dysfunction to severe decompensation requiring dialysis. Acute tubular necrosis (ATN) is the most common injury affecting renal tubular functions (3).

The need for better treatment strategies for ARI called for 
cellular based strategies aiming to regenerate damaged tissues. Stem cells are pleuripotential cells with the ability to initiate repair mechanisms improving organ protection and repair (4).

Mesenchymal stem cells (MSCs) are undifferentiated adult cells that can be isolated from a variety of tissues but primarily bone marrow stroma. The embryonic lineage of these cells is mesodermal. MSCs perform multiple functions: they act as a source for cellular replacement therapies, have immuno-modulatory and trophic activities, in addition to their paracrine functions. Thus, systemic delivery of MSCs induces both immuno-regulatory and regenerative trophic activities at sites of inflammation and tissue damage (5).

MSCs hold special promise in attenuating kidney injury, since nephrons are largely of mesenchymal origin. Furthermore, stromal cells are key players in signaling of differentiation of both nephrons and collecting ducts (4).

The objective of this study is to evaluate the role of mesenchymal stem cells in regeneration of post ischemic acute renal injury in mongrel dogs.

\section{Materials and Methods}

\subsection{Subjects}

Fifteen adult male mongrel dogs were used in this study. All animals received veterinary care in compliance with the 'Guide for the Care and Use of Laboratory Animals' and the procedures described were approved by the Institutional Ethics Committee. Animals were divided as follows:

Group I

Three adult healthy mongrel dogs weighing 15 to $20 \mathrm{~kg}$ subjected to bone marrow aspiration (normal group).

Group II

Nine adult mongrel dogs weighing 15 to $20 \mathrm{~kg}$ used as control undergoing acute renal injury by clamping both renal pedicles for 60 minutes and not subjected to stem cell therapy which were further divided into:

a. Three dogs without sham injection and scarified 3 days after IRI.

b. Three dogs without sham injection and scarified 7 days after IRI.

c. Three dogs with sham injection and scarified 7 days after IRI.

Group III

Three adult mongrel dogs weighing 15 to $20 \mathrm{~kg}$ used as control undergoing acute renal failure and then receiving systemic MSC injection.

\subsection{Induction of Acute Renal Injury Model (Ischaemia/Reperfusion Experiment)}

Animals were anesthesized with intramascular injection of ketamin $90 \mathrm{mg} / \mathrm{kg}$ and xylagect $10 \mathrm{mg} / \mathrm{kg}$ (Euthanex, Palmer, PA). Animals were kept on temperature-regulated pad to maintain their core temperature at $37^{\circ} \mathrm{C}$ throughout surgery. ARI was induced, using a transabdominal approach, by clamping of both renal pedicles for $60 \mathrm{~min}$ with nontraumatic microvascular clamps (Roboz Surgical Instrument Co, 630 E Diamond Ave, Gaithersburg, MD 20877).

Reperfusion was confirmed visually before the abdominal incision was closed, animals received $50 \mathrm{mg} / \mathrm{kg}$ of antibiotic daily. At the end of surgery all dogs received $100 \mathrm{ml}$ of saline intravenous to replace fluid loss.

\subsection{Stem Cell Preparation}

\subsubsection{Sampling}

15-20 $\mathrm{ml}$ bone marrow was aspirated from posterior iliac spine under general anaesthesia on preservative free heparin.

\subsubsection{MSC Separation}

Mononuclear cells (MNC) were separated using Ficolhypaque density gradient centrifugation. MNCs were plated in T25 flasks in a density of 1 million cells per flask in complete medium (DMEM, 20\% fetal calf serum, penicillin/streptomycin). After 3-5 days, non-adherent cells were discarded and the medium was replenished. When cells reached $80-85 \%$ confluence, cells were harvested using $0.25 \%$ trypsin-EDTA. Cells were washed, counted and subjected to:

1. Immunophenotyping

2. Trilineage differentiation (osteogenic, chondrgenic and adipogenic)

3. Viability testing

4. Iron oxide tagging using Ferridex (Bayer): using 50 micron iron oxide in 4ml RPMI media for 30 minutes followed by centrifugation at $2000 \mathrm{xg}$ for 10 minutes.

\subsubsection{MSC Infusion}

Animals were treated with approximately 2 million of MSCs per kilogram injected systemically through the femoral vein.

Evaluation of Therapeutic Effect:

Functional evaluation of renal functions using serum creatinine preoperative and 30 minutes, 1 day, 2days, 4days, 7days postoperative.

Renal histology and injury scores

After animals were sacrificed, the kidneys were harvested, fixed in $4 \%$ buffered formalin and embedded in paraffin wax. Coronal sections $(4 \mu \mathrm{m})$ were dehydrated using ethanol and were stained with haematoxylin-eosin, PAS and Prussian blue stain. Degree of renal injury after ischaemia was evaluated by two observers blind towards the group categories. Using a quantitative method, twenty randomly selected microscope fields per kidney (magnification X400) were evaluated and scored using a scale ranging from 0 to 4 .

Scoring:

The following items were recorded:

- Presence of focal necrosis,

- Inflammatory cell infiltration,

- Dilated glomerular chamber,

- Casts,

- Dilated tubules

- Abnormality of the glomerular tuft.

A score of 0 signified absence of lesions, and scores of 1, 2, 3 and 4 corresponded to $1-10 \%, 11-25 \%, 26-50 \%$ and $51-$ 
$100 \%$ involvement of the microscope field, respectively, for all items.

1. Tracing of injected MSCs (homing) in renal tissues using Prussian blue staining.

2. Immunohistochemical evaluation of the apoptosis and proliferative capacity of MSCs using caspase 3 and KI 67.

3. Morphometrical studies:

The renal injury induced by ischemia (dilated glomerular chamber, dilated tubules, epithelial shedding and necrosis) and the effect of the MSCs was evaluated using computer based image analysis system. Statistical analysis of morphometric results was done by ANOVA test, Bonferroni, Post Hoc test, NPar Tests, Kruskal-Wallis Test and MannWhitney Test.

Statistical Methods:

Statistical analysis was done SPSS 15.0.1 for windows (SPSS Inc, Chicago, IL, 2001).

\section{Results}

\subsection{Evaluation of Renal Injury}

Renal function tests done showed the successful induction of acute renal failure. Follow up of renal function tests showed gradual improvement (Fig. 1).
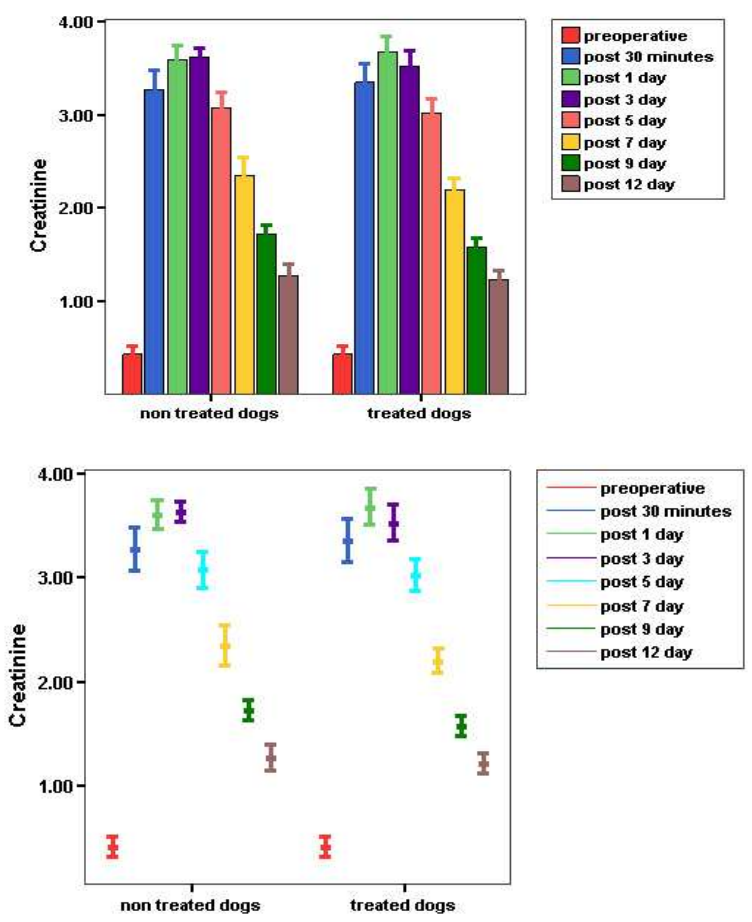

Fig. 1. Histogram represents comparison between serum creatinine in ischemic non treated and in MSCs treated dogs.

\subsubsection{Mesenchymal Stem Cells Identification}

MSCs were identified according to morphology, the ability for trilineage differentiation and flowcytometric analysis (Fig. 2). Mesenchymal stem cells flow cytometric analysis. MSCs were positive for CD44 and CD105 whereas being negative for CD31, CD34 CD45. Fig. 2:

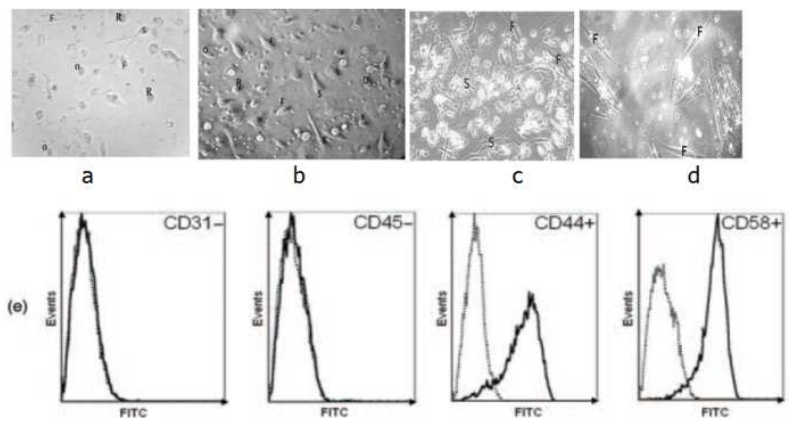

Fig. 2. MSC identification: A-D Sequential morphological evaluation of MSCs. E. Flowcytometric analysis.

\subsubsection{The Role of MSCs in Post Ischemic Acute Renal Injury}

Histological Results (H\&E and PAS stains):

In IRI groups, renal lesions consisted mainly of focal acute tubular necrosis and cortical necrosis. Acute tubular necrosis ranged from dilated tubules to more important lesions, such as degeneration and frank necrosis of tubular segments. Both acute tubular necrosis and cortical necrosis were found in most sections of ischemic groups with variable extent.

Group IIa: Untreated IRI at day3

Examination of sections in the renal cortex and outer medulla of dogs belonging to this subgroup showed the presence of focal necrosis of tubular segments with destructed basement membrane and intercellular wall, inflammatory cell infiltration, dilated glomerular chamber (widening of Bowman's space), glomerular fissuring, congestion in peri tubular capillaries, vacuolated cytoplasm, shedding of epithelial lining with cast formation, dilated tubules and dark pyknotic nuclei (Fig 3).

Group IIb: Untreated IRI at day 7

Sections in the renal cortex an outer medulla of dogs belonging to this subgroup showed diminished focal necrosis of tubular segments, decreased plethora in peri tubular capillaries, less vacuolated cytoplasm, cast formation, persistance of inflammatory cell infiltration, dilated glomerular chamber, glomerular fissuring, dilated tubules with mild destructed BM and dark pyknotic nuclei (fig. 3)

Group IIc: IRI with sham injection at day 7

Sections in the renal cortex and outer medulla of dogs belonging to this subgroup showed the same findings as group II b, diminished of focal necrosis of tubular segments, decreased congestion in peri tubular capillaries, less vacuolated cytoplasm, cast formation, persistance of inflammatory cell infiltration, dilated glomerular chamber, glomerular fissuring, dilated tubules with mild destructed BM and dark pyknotic nuclei (fig.3).

Group III: IRI with immediate MSC injection at day 7

Ischemic renal lesions did not improve by MSC treatment, there was no changes in the progression of course of IRI. All kidneys showed morphological features of acute renal lesions in group IIb, IIc consisting mainly of diminished focal necrosis of tubular segments, decreased congestion in peritubular capillaries, less vacuolated cytoplasm, cast formation, persistence of inflammatory cell infiltration, 
dilated glomerular chamber, glomerular fissuring, dilated tubules with mild destructed BM and dark pyknotic nuclei

(fig.3).

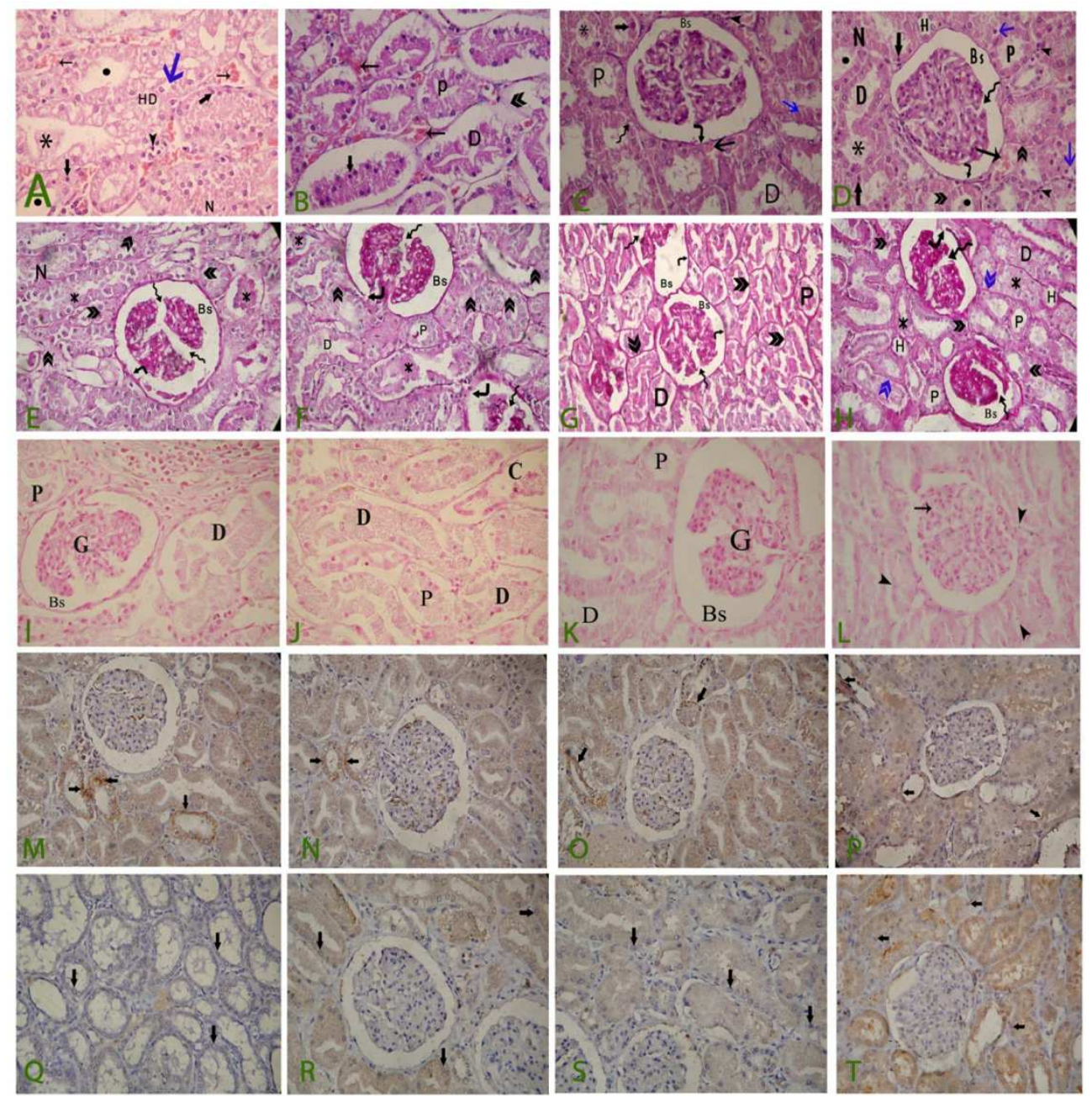

Fig. 3. Histological evaluation of stem cell effect

(A-D)H \& E sections showing morphological changes in examined groups (400X)

A. Group IIa: Photomicrograph of a renal outer medulla of dog belonging to group IIa showing presence of focal necrosis of tubular segments $(\mathrm{N})$, area of hydropic degeneration (HD), inflammatory cell infiltration (arrow head >), congestion in peritubular capillaries (thin black arrow $\rightarrow$ ), Vacuolated cytoplasm (green arrow $\rightarrow$ ), dilated tubules $(\bullet)$ with shedding of epithelial lining $(\boldsymbol{\nabla})$ and dark pyknotic nuclei (thick black arrow $\downarrow$ ).

B. Group IIb: Photomicrograph of a renal outer medulla of dog belonging to group IIb showing diminished focal necrosis of tubular segments, swollen narrowed proximal (P) tubules and wide sloughed distal (D) tubules with mild destructed basement membrane (duple arrow head »), congested peritubular capillaries (thin black arrow $\rightarrow$ ) and detached pyknotic nuclei (thick black arrow $\downarrow$ ).

C. Group IIc: Photomicrograph of a renal cortex and outer medulla of dog belonging to group IIc showing the same findings as Group II $b$, diminished focal necrosis of tubular segments, decreased plethora in peritubular capillaries (thin black arrow $\rightarrow$ ), less vacuolated cytoplasm (thin green arrow $\rightarrow$ ), cast formation ( ), persistence of inflammatory cell infiltration(arrow head $\triangleright$ ), dilated bowman's space (Bs), glomerular fissuring (tortuous black arrow $\rightarrow$ ), dilated proximal (P) and distal (D) tubules $(\bullet)$ with intact bowman's capsule (curved arrow $\curvearrowright$ ) and basal pyknotic nuclei (thick black arrow $\rightarrow$ ).

D. Photomicrograph of a renal cortex and outer medulla of dog belonging to group III showing the diminished of focal necrosis of tubular segments $(\mathrm{N})$, tubular dilatation $(\bullet)$ of proximal (P), distal (D) convoluted tubules and loop of henl $(\mathrm{H})$, decreased congestion in peritubular capillaries (thin black arrow $\rightarrow$ ), with persistence of inflammatory cell infiltration (arrow head >). Renal tubules show minimal epithelial shedding and cast formation ( ), the epithelium has basal less pyknotic nuclei (thick black arrow $\downarrow$ )with minimal vacuolated cytoplasm (thin green arrow $\rightarrow$ ) and intact basement membrane (»). The glomerular fissuring (tortuous arrow $\sim$ ) was diminished within dilated Bowman's space (Bs) and intact Bowman's capsule $(\curvearrowright)$

(E-H) PAS stained sections showing morphological 
changes in examined groups (400X)

E Photomicrograph of a renal cortex and outer medulla of dog belonging to group IIa showing A) presence of focal necrosis of tubular segments $(\mathrm{N})$ with destructed basement membrane (») and intercellular wall, widening of Bowman's space (Bs) with destructed Bowman's capsule ( $\curvearrowleft$ ), glomerular fissuring $(\sim)$ and shedding of epithelial lining with cast formation ( ). B) Destructed basement membrane (») of renal tubules, proximal (P), distal (D) convoluted tubules and loop of henl $(\mathrm{H})$ with cast formation ( )

F. Photomicrograph of a renal cortex and outer medulla of dog belonging to group IIb showing diminished focal necrosis of tubular segments, persistence of dilated glomerular chamber (widening of Bowman's space) (Bs) with minimal destructed Bowman's capsule (curved arrow $\curvearrowleft$ ), glomerular fissuring (tortuous arrow $\sim$ ), swollen proximal $(\mathrm{P})$ convoluted tubules and distal (D) convoluted tubules with less destructed basement membrane (duple arrow head $\gg$ ) and minimal cast formation ( )

G. Photomicrograph of a renal cortex of dog belonging to group IIc showing the same findings as Group IIb, improvement in the epithelial lining of proximal $(\mathrm{P})$ and distal (D) convoluted tubules with mild destructed basement membrane (»)). Persistence of dilated glomerular chamber (widening of Bowman's space) ( Bs) with minimal destructed Bowman's capsule (curved arrow $\curvearrowleft$ ), glomerular fissuring (tortuous arrow $\sim$ ),

H. Photomicrograph of a renal cortex and outer medulla of dog belonging to group III showing tubular dilatation of proximal (P), distal (D) convoluted tubules and loop of henle (H). Renal tubules show minimal epithelial shedding and cast formation ( ), intact basement membrane (green $\gg$ ), with destruction in some areas (»). The glomerular fissuring (tortuous arrow $\sim$ ) demonstrated within dilated glomerular chamber (Bs), with intact or destructed Bowman's capsule $(\curvearrowright)$.

(I-L) Prussian blue stained sections showing MSC tracking in examined groups (400X):

Positive cells were seen at the epithelial lining of the cortical tubules, the glomeruli, and at the Bowman's space. The renal cortex and outer medulla showed multiple Prussian blue positive cells among the epithelial lining of the CT and blood vessels
I. Group IIa
J. Group IIb
K. Group IIc
L. Group III
(M-P) Caspase Positivity in studied groups (400X):

M. Photomicrograph of a renal cortex and outer medulla of (ischemic) dog group IIa showing caspase 3 positive tubules were observed (black arrow $\rightarrow$ ).

N. Photomicrograph of a renal cortex and outer medulla of (ischemic) dog group IIb showing caspase 3 positive tubules were observed (black arrow $\rightarrow$ ).

O. Photomicrograph of a renal cortex and outer medulla of (ischemic) dog group IIc showing caspase 3 positive tubules were observed (black arrow $\rightarrow$ ).
P. Photomicrograph of A) renal cortex and B) outer medulla of treated dog group III showing caspase 3 positive tubules were observed (thin black arrow $\rightarrow$ ).

(Q-T) Ki67 Positivity in studied groups (400X):

Q. Photomicrograph of a renal cortex and outer medulla of (ischemic) dog group IIa showing negative staining KI 67 cells were observed (black arrow $\rightarrow$ ).

R. Photomicrograph of a renal cortex and outer medulla of (ischemic) dog group IIb showing negative staining KI 67 cells were observed (black arrow $\rightarrow$ ).

S. Photomicrograph of a renal cortex and outer medulla of (ischemic) dog group IIc showing negative staining KI 67 cells were observed (black arrow $\rightarrow$ ).

T. Photomicrograph of A) renal cortex and B) outer medulla of treated dogs group III showing negative staining KI 67 cells were observed (black arrow $\rightarrow$ ).

Statistical evaluation showed that renal lesion score of MSC treated group III $(1.12 \pm 0.36)$ was not significantly different from the score of the non treated group IIb, IIc (score of $1.17 \pm 0.42$ ). In IRI MSC-treated group III, infused MSCs did not trigger vasculature thrombosis nor enhance glomerular or tubular lesions when compared to IRI non treated group IIb, IIc..

Prussian Blue Stained Sections:

At day 7 after MSC transplantation, all kidneys were evaluated to assess MSC localization. Positive cells were seen at the epithelial lining of the cortical tubules, there were Prussian blue positive cells in the glomeruli, and at the Bowman's space. The renal cortex and outer medulla showed multiple Prussian blue positive cells among the epithelial lining of the CT and blood vessels (Fig.3 I-L).

\section{Caspase 3 Immunostained Sections:}

At day 7 after MSC transplantation, all kidneys were evaluated to assess apoptosis by caspase 3 positive tubules. Caspase 3 shows cytoplasmic staining. The control dogs appeared caspase 3 negative tubules. In the (ischemicoperated) dog group, caspase 3 positive tubules were observed $(1.55 \pm 1.80)$. Results showed that there was no significant increase in cell apoptosis in large animals in groups submitted to IRI when compared to the MSC-treated IRI dog group. There were approximately (1.42 \pm 1.39$)$ caspase 3 positive tubules (Fig.3 M-P).

\section{KI 67 Immunostained Sections:}

At day 7 after MSC transplantation, all kidneys were evaluated to assess proliferation by KI 67 positive cells. KI 67 shows nuclear staining. The control dogs appear KI 67 negative tubules. In the (ischemic-operated) dog group, KI 67 positive cells were observed $(0.97 \pm 1.40)$. Results showed that there was no significant increase in cell proliferation in large animals in groups submitted to IRI when compared to the MSC-treated IRI dog group. There were approximately $(1.39 \pm 2.19) \mathrm{KI} 67$ positive cells (Fig.3 Q-T).

\section{Morphometry:}

A. Morphometry on $\mathrm{H} \& \mathrm{E}$ sections by the application of One way ANOVA test revealed the following results:

1. Glomerular diameter:

The mean glomerular area was $(1207.04 \pm 119.93)$ in 
normal dog (Group I), (1632.44 \pm 120.19$)$ in ischemic non treated (Group II) and (1349.50 \pm 150.53$)$ in MSCs treated group (Group III) (Table 1). Statistical analysis revealed that, although the diameter was less in treated than non-treated dogs, the difference was not statistically significant; while the difference between normal and ischemic dogs was statically significant $(\mathrm{P}<0.05)$ (Table 1 , Fig. 4).

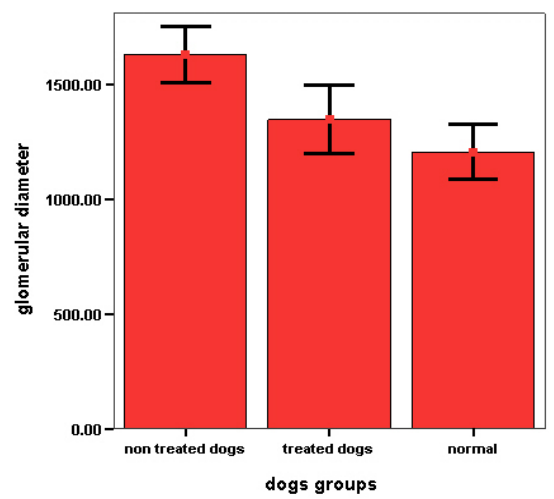

Fig. 4. Histogram represents the percentage of glomerular diameter in normal dog, ischemic non treated and in MSCs treated dogs.

Table 1. Comparison between non treated, treated and normal (control) dogs in statistical parameters of necrosis, tubular dilatation, epithelial shedding and glomerular diameter by One way ANOVA test.

\begin{tabular}{llllll}
\hline dogs groups & & Necrosis & dilatation & epithelial shedding & glomerular diameter \\
\hline \multirow{4}{*}{ non treated dogsd } & N & 9.00 & 9.00 & 9.00 & 9.00 \\
& Mean & 10.44 & 1093.78 & 2.00 & 1632.44 \\
& Std. deviation & 1.33 & 237.81 & 0.87 & 120.19 \\
& Std. Error of mean & 0.44 & 79.27 & 0.29 & 40.06 \\
& Median & 10.00 & 1060.00 & 2.00 & 1600.00 \\
& Minimum & 8.00 & 800.00 & 1.00 & 1500.00 \\
& Maximum & 12.00 & 1444.00 & 3.00 & 1900.00 \\
N & 9.00 & 9.00 & 9.00 & 1349.50 \\
Mreated dogs & 6.11 & 847.78 & 2.11 & 150.53 \\
& Std. deviation & 1.76 & 228.98 & 0.78 & 50.18 \\
& Std. Error of mean & 0.59 & 76.33 & 26.00 & 1309.00 \\
& Median & 7.00 & 844.00 & 2.00 & 1167.31 \\
& Minimum & 3.00 & 450.00 & 1.00 & 1613.50 \\
& Maximum & 8.00 & 1200.00 & 3.00 & 9.00 \\
Normal & N & 9.00 & 9.00 & 9.00 & 1207.04 \\
& Mean & 1.11 & 733.16 & 0.56 & 119.93 \\
& Std. deviation & 0.78 & 121.89 & 0.53 & 39.98 \\
& Std. Error of mean & 0.26 & 40.63 & 0.18 & 1223.00 \\
& Median & 1.00 & 795.57 & 1.00 & 1012.63 \\
& Minimum & 0.00 & 505.00 & 0.00 & 1363.84 \\
\hline
\end{tabular}

\section{Tubular Dilatation}

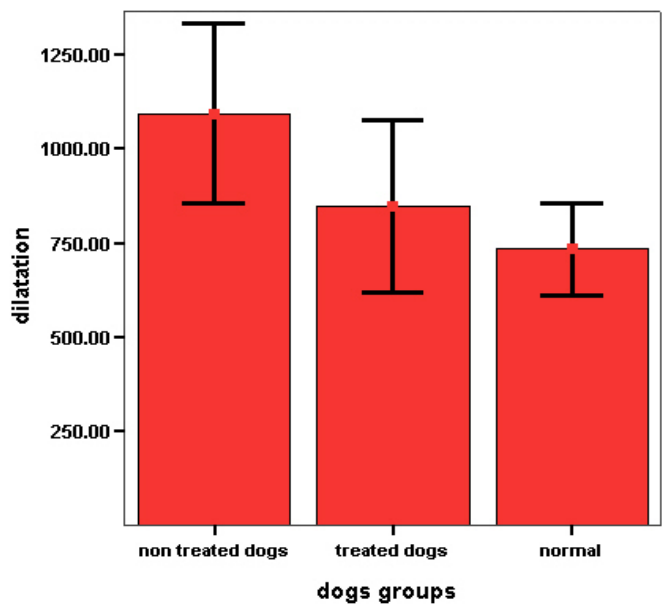

Fig. 5. Histogram represents the percentage of tubular dilatation in normal dog, ischemic non treated and in MSCs treated dogs.
Table 2. Comparison between non treated, treated and normal (control) dogs in statistical significance ( $P$ value) of tubular dilatation and glomerular diameter by Post Hoc test.

\begin{tabular}{llll}
\hline Depentent variable & (I) dogs groups & $(\mathbf{J})$ dogs groups & Sig. \\
& Non treated dogs & treated doghs & 0.051 \\
& & normal & 0.003 \\
& treated dogs & non treated dogs & 0.051 \\
& & normal & 0.073 \\
& \multirow{2}{*}{ normal } & non treated dogs & 0.003 \\
& & treated doghs & 0.729 \\
glomerular diameter & \multirow{2}{*}{ non treated dogs } & treated doghs & 0.000 \\
& & normal & 0.000 \\
& \multirow{2}{*}{ treated dogs } & non treated dogs & 0.000 \\
& & normal & 0.090 \\
& \multirow{2}{*}{ normal } & non treated dogs & 0.000 \\
& & treated doghs & 0.090 \\
\hline
\end{tabular}

The mean tubular dilatation was $(733.16 \pm 121.89)$ in normal dog (Group I), (1093.78 \pm 237.81$)$ in ischemic non treated (Group II) and $(847.78 \pm 228.98)$ in MSCs treated 
group (Group III) (Table 1). Statistical analysis revealed significant dilatation in ischemic $\operatorname{dogs}(\mathrm{p}<0.05)$ while there was an improvement in the treated group which was not statistically significant $(\mathrm{P}>0.05)$ (Table 2, Fig. 5).

3. Epithelial shedding:

Mean count of tubules with epithelial shedding in haematoxylin and eosin stained sections was $(0.56 \pm 0.53)$ in normal dog (Group I), $(2.00 \pm 0.87)$ in ischemic non treated (Group II) and $(2.11 \pm 0.78)$ in MSCs treated group (Group III) (Table 1). Statistical analysis revealed no effect of MSCs on treated as compared with non-treated dogs (group and MSCs treated $(\mathrm{P}<0.05)$ (Table 3, Fig. 10).

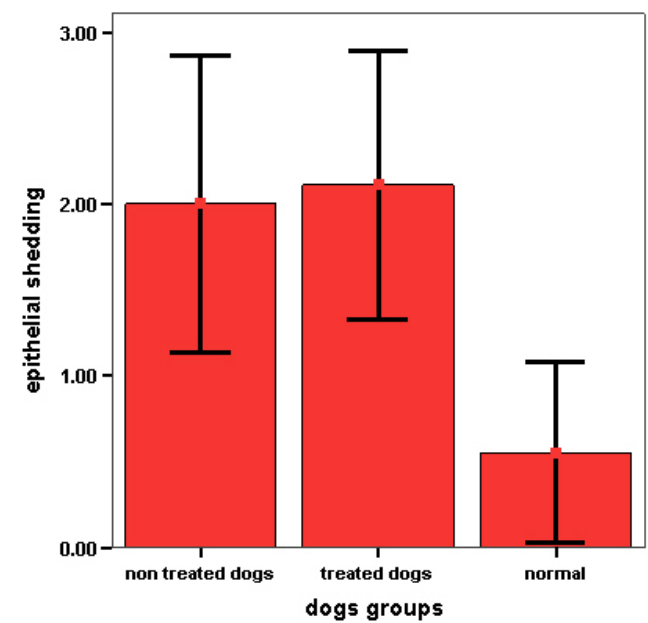

Fig. 6. Histogram represents the percentage of epithelial shedding in normal dog, ischemic non treated and in MSCs treated dogs.

Table 3. Comparison between non treated, treated and normal (control) dogs in statistical significance ( $P$ value) of necrosis and epithelial shedding by Post Hoc test.

\begin{tabular}{lllll}
\hline animal & groups & & K1 67 & Caspase 3 \\
\hline \multirow{3}{*}{ control } & $\mathrm{N}$ & 33.00 & 33.00 \\
& Mean & 0.97 & 1.55 \\
& Std. deviation & 1.40 & 1.80 \\
& Median & 0.00 & 1.00 \\
$\operatorname{dog}$ & Minimum & 0.00 & 0.00 \\
& Maximum & 5.00 & 6.00 \\
& N & 33.00 & 33.00 \\
& Mean & 1.39 & 1.42 \\
& treated & Std. deviation & 2.19 & 1.39 \\
& & Median & 0.00 & 1.00 \\
& & Minimum & 0.00 & 0.00 \\
& & Maximum & 9.00 & 4.00 \\
\hline
\end{tabular}

\section{Necrosis}

Mean tubular showed the tubular necrosis was $(1.11 \pm 0.78)$ in normal $\operatorname{dog}$ (Group I), (10.44 \pm 1.33$)$ in ischemic non treated (Group II) and (6.11 \pm 1.67$)$ in MSCs treated group (Group III) (Table 1). Statistical analysis revealed a significant difference of MSCs on treated dog corresponding to ischemic non treated group and normal dog (significant difference $\mathrm{P}<0.05$ ) (Table 3, Fig. 7).

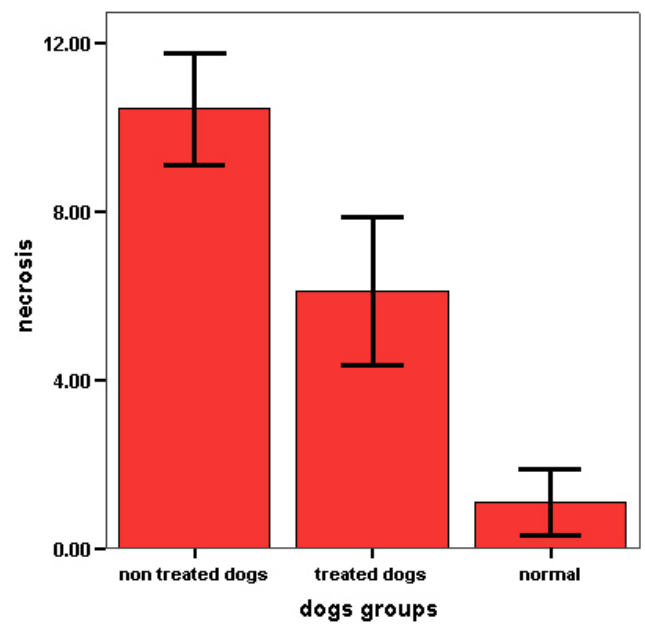

Fig. 7. Histogram represents the percentage of necrosis in normal dog, ischemic non treated and in MSCs treated dogs

\section{B. Morphometry on Immunostained sections}

\section{Caspase 3 Immunostained Sections}

At day 7 after MSCs transplantation, all kidneys were evaluated by the application of NPar Tests and MannWhitney Test to assess apoptosis by caspase 3 positive tubules (dark brown cytoplasm). The number of positive tubules was $(1.55 \pm 1.80)$ in ischemic non treated (Group II) and $(1.42 \pm 1.39)$ in MSCs treated group (Group III) (Table 4). This difference was not statistically $(\mathrm{P}-$ value $=0.91)$ (Table 4) (Fig. 8).
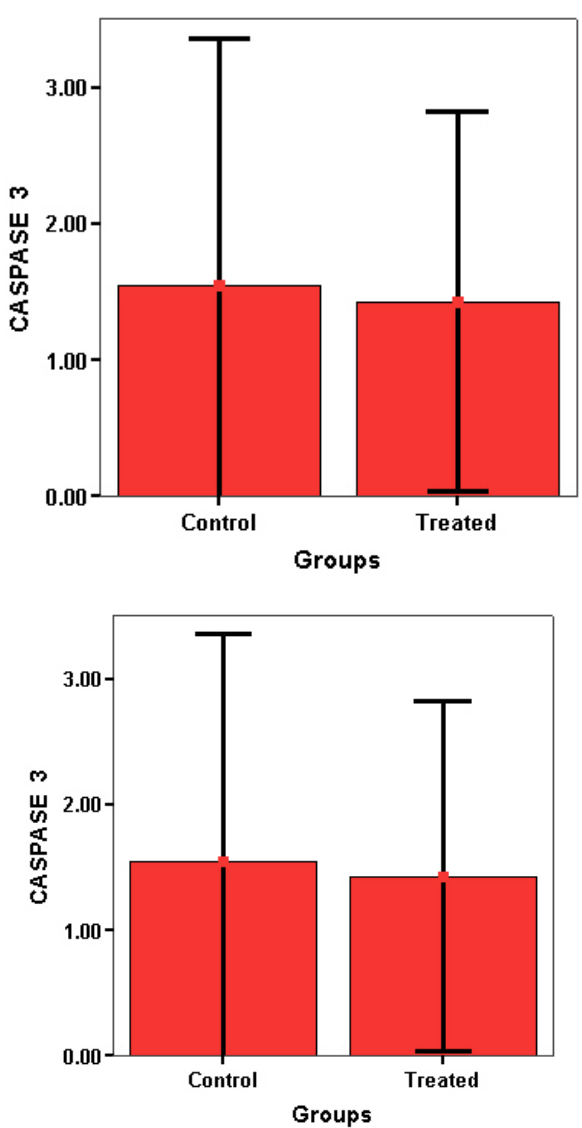

Fig. 8. Histogram represents the percentage of caspase 3 positive tubules in ischemic non treated and in MSCs treated dogs. 
Table 4. Comparison between treated and normal (control) dogs regards percentage of positive cells and tubules of KI 67 and Caspase 3 by NPar and MannWhitney test.

\begin{tabular}{llll}
\hline animal & & K1 67 & Caspase 3 \\
\hline \multirow{3}{*}{$\operatorname{dog}$} & Mann-whitney U & 520.000 & 536.500 \\
& Z & -0.345 & -0.107 \\
& p-value & 0.730 & 0.915 \\
\hline
\end{tabular}

Table 5. Comparison between treated and normal (control) dogs regards statistical significance (P value) of percentage of positive cells and tubules in KI 67 and Caspase 3 by NPar and Mann-Whitney test.

\begin{tabular}{|c|c|c|c|c|c|c|c|}
\hline groups & & pre operative creatinine & post 30 minut & post 1 day & post 3 day & post 5 day & post 7 day \\
\hline \multirow{7}{*}{ non treated dogs } & $\mathrm{N}$ & 4.00 & 4.00 & 4.00 & 4.00 & 4.00 & 4.00 \\
\hline & Mean & 0.43 & 3.28 & 3.60 & 3.63 & 3.08 & 2.35 \\
\hline & Std. deviation & 0.10 & 0.21 & 0.14 & 0.10 & 0.17 & 0.19 \\
\hline & Std. Error of mean & 0.05 & 0.10 & 0.07 & 0.05 & 0.09 & 0.10 \\
\hline & Median & 0.45 & 3.25 & 3.55 & 3.65 & 3.05 & 2.40 \\
\hline & Minimum & 0.30 & 3.10 & 3.50 & 3.50 & 2.90 & 2.10 \\
\hline & Maximum & 0.50 & 3.50 & 3.80 & 3.70 & 3.30 & 2.50 \\
\hline \multirow{7}{*}{ treated dogs } & $\mathrm{N}$ & 4.00 & 4.00 & 4.00 & 4.00 & 4.00 & 4.00 \\
\hline & Mean & 0.43 & 3.35 & 3.68 & 3.53 & 3.03 & 2.20 \\
\hline & Std. deviation & 0.10 & 0.21 & 0.17 & 0.17 & 0.15 & 0.12 \\
\hline & Std. Error of mean & 0.05 & 0.10 & 0.09 & 0.09 & 0.08 & 0.60 \\
\hline & Median & 0.05 & 3.35 & 3.65 & 3.55 & 3.00 & 2.20 \\
\hline & Minimum & 0.30 & 3.10 & 3.50 & 3.30 & 2.90 & 2.10 \\
\hline & Maximum & 0.50 & 3.60 & 3.90 & 3.70 & 3.20 & 2.30 \\
\hline
\end{tabular}

KI 67 Immunostained Sections:

At day 7 after MSCs transplantation, all kidneys were evaluated by the application of NPar Tests and MannWhitney Test to assess proliferation by KI 67 positive cells (dark brown nucleus). In the control ischemic-operated dog (Group II), KI 67 positive cells were observed $(0.97 \pm 1.40)$ and $(1.39 \pm 2.19) \mathrm{KI} 67$ positive cells in MSCs treated group (Group III) (Table 4). Although there was an increase in cellular proliferation in the treated group, this difference was not statistically significant group $(\mathrm{P}-$ value $=0.730)($ Table 5$)$ (Fig. 9).

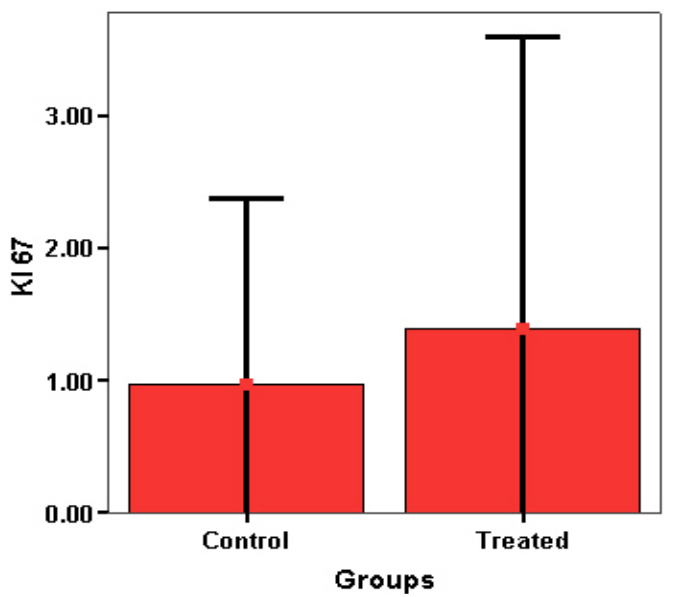

Fig. 9. Histogram represents the percentage of KI 67 positive cells in ischemic non treated and in MSCs treated dogs.

\section{Discussion}

Ischemic reperfusion injury is a clinically significant problem in renal transplantation. Renal I/R injury in the early transplant period has been associated with acute rejection, delayed graft function, and late allograft failure (6)

Mesenchymal stem cells of all bone marrow derived cells, hold special promise in attenuating kidney injury, since nephrons are largely of mesenchymal origin and stromal cells are of crucial importance for signaling leading to differentiation of both nephrons and collecting ducts (4).

To the best of our knowledge, this is the first time that the effect of MSC transplantation in post ischemic acute renal injury has been evaluated in mongrel dog. In our experimental setting, autologous MSCs localized to the kidney, but did not provide any beneficial effect on the course of the ischaemia-reperfusion injury in mongrel dog.

In the present work, our experiments in dogs a model for large animal has much values, the dog represents a suitable model for the study of human diseases. The physiology, disease presentation and clinical responses of the dog are much more similar to man when compared with various other traditional animal models.

In the present study, canine model for ARF was done through ischemia reperfusion experiment. Behr et al (2009) found that $60 \mathrm{~min}$. bilateral ischemia induced mild renal injury with focal acute tubular necrosis; this pattern of lesions is closer to that observed in humans with ARF (7). 
In the present work, allogenic MSC transplantation was used. (3) Morigi et al (2004) demonstrated the superiority of MSCs over haematopoietic stem cells (HSCs) in a cisplatin induced model of renal damage (3) and studies done by (8) Tögel et al. (2005), (9) Herrera et al. (2007) and (10) Semedo et al. (2007) have used MSCs that proved efficient in experimental ARF models (8-10).

The route of stem cell injection differs among experimental studies: intravenous done by Morigi et al. (2004) (3), and Imberti et al. (2007) (11), intra-arterial done by Lange et al. (2005 (12) and Tögel et al. (2007) (13) and intraperitoneal done by $\mathrm{Bi}$ et al. (2007)(14) methods of infusion have been reported. We have chosen the intravenous route that has been successfully used by Morigi et al. (2004) (3) and Imberti et al. (2007)(11), in order to deliver stem cells to the target organ more efficiently than intraperitoneal. Although intra-arterial is the best of delivering but the practical performance is more difficult.

In this study, cell tracking was done through labeling of cells with iron-oxide which has been shown to be nontoxic and long-lasting method for in vivo tracking of cells, including stem cells. We demonstrate here that iron-oxide labeled mesenchymal stem cells injected after ARF can be histologically located in the kidney.

Behr et al (2009) examined the kidneys at day 7 after MSC transplantation to assess MSC localization. MSC were found only in the renal cortex and not in the medulla. In the cortex, MSCs were found in glomeruli and were rarely identified in the renal microvasculature. The MSC was not found in renal tubules (7).

Initial studies done by Morigi et al. (2004) (3), Lin et al. (2003) (15) and Imasawa et al. (2001) (16) in different models of acute renal failure have suggested that MSCs engrafted in the damaged kidney and differentiated into tubular epithelial cells. Since then, other studies done by Tögel et al. (2007)(13) and Lange et al. (2005)(12) have found that MSCs do not engraft into tubules, labeled MSCs being detected mostly in glomeruli, still ameliorating renal function.

However, the absence of MSCs in the tubules in the work of Behr et al (2007) (7) was one week after injection, kidneys evaluated 3 weeks after MSC injection, showed presence of MSCs both in glomeruli and in tubules. Moreover, the cells had acquired markers of renal cells in glomeruli as well as in tubules, suggesting they underwent trans-differentiation.

Herreraet al (2007)(9) proved that after 24 hours, injected MSCs were mainly detectable within peritubular capillaries and interstitium, whereas after 8 days MSCs were found mainly in tubules. Broekema et (2005)(17) showed that bone marrow derived cells engraftment in tubules was optimal on day 14, decreasing by day 28 after the ischaemic insult which disagree with our results.

In the present work, as expected, IRI was followed by significant increase in serum creatinine, consequences of renal vasoconstriction, reduced glomerular filtration rate and tubular injury secondary to renal ischaemia. No significant differences was observed in serum creatinine in MSC treated dogs when compared with non treated dogs or change the course of renal IRI.

Behr et al. (2009)(7) found that in sheep model of IRI was followed by significant increase in plasma renin activity, creatinine/ BUN concentrations, and urinary sodium excretion, consequences of renal vasoconstriction, reduced glomerular filtration rate and tubular injury secondary to renal ischaemia. No significant difference in these parameters was observed after MSCs infusion during the 7-day period following our IRI. However, the mechanism of such a glomerular filtration rate improvement has not been defined; an effect on total renal outer cortical blood flow or renovascular resistance was not found.

In the present work, the kidney tissues of ischemic non treated animals (group IIa) showed the features of acute tubular necrosis in form of focal necrosis of tubular segments with destructed basement membrane and intercellular wall, inflammatory cell infiltration, congestion in peri-tubular capillaries, vacuolated cytoplasm, shedding of epithelial lining with cast formation, dilated tubules, dark pyknotic nuclei and dilated glomerular chamber with glomerular fissuring. The latter findings were confirmed by a significant increase in serum creatinine from base line to $2-3$ folds and increase cellular apoptosis which demonstrated by the presence of caspase 3 positive cells. On the contrary group $\mathrm{IIb}, \mathrm{c}$ showed improvement of renal injury which is confirmed by a significant decrease in serum creatinine to base line in 7 days and decrease cellular apoptosis which was demonstrated by the diminished caspase 3 positive cells.

Thadhani et al (1996) (19) Bonventre. (1993) (20), Sutton and Molitoris (1998) (21) found that the proximal tubules (S3 segments) located in the outer strip of the outer medulla are highly sensitive to ischemic injury and responsible for the pathophysiological and clinical presentation of ARF. Kitada et al (2002)(22), reported the histological examination At 72 hours post ischemic reperfusion injury, renal tissues in the ischemic animals showed moderate damage of tubular cells, whereas the damage was slight in the treated animals.

Scheiermann et al (2009)(23) studied the pathogenesis of $\mathrm{I} / \mathrm{R}$ injury and described a central role of inflammatory mediators. Jang et al, (2009) (24) suggested that the immune modulators in the early phase of AKI triggers activation of kidney dendritic cells leading to attraction of an array of leukocytes.

In the present work, the kidney tissues of ischemic treated dogs (group III) showed mild, although statistically nonsignificant improvement by MSC treatment. However, there were no changes in the progression of course of IRI. This was probably due to the fact that even non-treated animals showed improvement, suggesting a higher regenerative capacity of the canine kidneys.

All kidneys showed morphological features of healed acute renal lesions in group IIb, IIc consisting mainly of diminished focal necrosis of tubular segments, decreased congestion in peri-tubular capillaries, less vacuolated cytoplasm, cast formation, persistance of inflammatory cell infiltration, dilated glomerular chamber, glomerular fissuring, 
dilated tubules with mild destructed basement membrane and dark pyknotic nuclei. The latter findings were confirmed by a significant decrease in serum creatinine to base line in 7 days

Homing of MSCs to kidney tissues was demonstrated by multiple Prussian blue positive cells seen at the epithelial lining of the cortical tubules, glomeruli, and at the Bowman's space without transdifferentiations to renal cells

At the cellular level decrease cellular apoptosis was demonstrated by diminished caspase 3 positive cells. However, there was no significant increase in cell proliferation in studied animals submitted to IRI when compared to the MSC-treated IRI dog group demonstrated by KI 67 stain.

These results agree with these of Behr et al (2009) (7) in large animal's model were no significant difference in these parameters was observed after MSCs infusion during the 7day period following our IRI. However, the mechanism of such a glomerular filtration rate (GFR) improvement has not been defined; an effect on total renal outer cortical blood flow or renovascular resistance was not found.

Humphreys and Bonventre, (2008) (25) mention that MSCs can secrete a broad array of growth factors in addition to cytokines, including granulocyte-colony stimulating factor, stem cell factor, leukemia-inhibitory factor, macrophagecolony stimulating factor, IL- 6, and IL-11. Recent studies have identified other growth factors and chemokines secreted by MSCs, such as vascular endothelial growth factor (VEGF), basic fibroblast growth factor, monocyte-chemoattractant protein-1, HGF, and insulin-like growth factor-1 (IGF-1).

Behr et al., (2009) (7) investigated the large-animal model at the molecular level. In the course of IRI, modification in expression of different molecules involved in inflammatory (TNF, IL-10), apoptotic (Bcl-2, caspase) and cell growth/ proliferation (VEGF). When investigating cell proliferation and apoptosis, they observed a significant increase in both phenomena after IRI, but again, no modulation in cell proliferation or death was found after MSC transplantation.

Different studies have shown that MSC infusion was associated with modification of expression of different molecules, some of them showing anti-inflammatory or vasculotropic properties $(8,13,11,10)$.

In conclusion, renal ischemic reperfusion (I/R) injury is a clinically significant problem in renal transplantation. Renal $\mathrm{I} / \mathrm{R}$ injury in the early transplant period has been associated with acute rejection, delayed graft function, and late allograft failure.

In the present work, we have shown that bone marrow derived MSCs can be detected in the renal cortex and medulla of treated animals. MSCs were seen at the epithelial lining of the cortical tubules, glomeruli, the Bowman's space, in some peri-tubular capillaries and among the epithelial lining of the renal tubules without transdifferentiation to renal cells. We conclude that their homing in these tissues, if coupled with targeting by cytokines or drugs, holds substantial promise for the development of a novel, cellbased approach to the treatment of clinical ARF. At any rate, integration of stem cells into the kidney may not be the key to improvement, and the beneficial effect of targeted MSC therapy may be a great promise.

\section{References}

[1] Cruz DN., Bagshaw SM., Ronco C. and Ricci Z. (2010): Acute kidney injury; classification and staging. Contrib. Nephrol., 164, 24-32.

[2] Morigi M., Imberti B., Zoja C., Corna D., Tomasoni S., Abbate M., Rottoli D., Angioletti S., Benigni A., Perico N., Alison M. and Remuzzi G. (2004): Mesenchymal stem cells are renotropic, helping to repair the kidney and improve function in acute renal failure. J. Am. Soc. Nephrol., 15:17941804.

[3] Kellum JA. (2008): Acute kidney injury. Crit. Care. Med., 36(4): 141-145.

[4] De Vries DK., Schaapherder AF. and Reinders ME. (2012): Mesenchymal stromal cells in renal ischemia/reperfusion injury. Frontiers in Immunology, 3 (162): 37-46.

[5] Bohle A., Christensen J., Kokot F., Osswald H., Schubert B., Kendziorra H., Pressler H. and Marcovic-Lipkovski J. (1990): Acute renal failure in man: new aspects concerning pathogenesis: a morphometric study. Am. J. Nephrol., 10: 374-388.

[6] Hiroyoshi T., Tsuchida M., Uchiyama K., Fujikawa K., Komatsu T., Kanaoka Y. and Matsuyama H. (2012): Splenectomy protects the kidneys against ischemic reperfusion injury in the rat. Transplant Immunology, 27 (1): $8-11$.

[7] Behr L., Hekmati M., Lucchini A., Houcinet K., Faussat AM., Borenstein N., Noel LH., Lelievre-Pegorier M. and Laborde K. (2009): Evaluation of the effect of autologous mesenchymal stem cell injection in a large-animal model of bilateral kidney ischaemia reperfusion injury. Cell Prolif., 42: 284 - 297.

[8] Tögel F., Hu Z., Weiss K., Isaac J., Lange C. and Westenfelder C. (2005): Administered mesenchymal stem cells protect against ischemic acute renal failure through differentiationindependent mechanisms. Am. J. Physiol. Renal Physiol., 289 (1): F31-F42.

[9] Herrera MB., Bussolati B., Bruno S., Morando L., Mauriello L., Romanazzi G. and Sanavio F. (2007): Exogenous mesenchymal stem cells localize to the kidney by means of CD44 following acute tubular injury. Kid. Int., 72: 430-441.

[10] Semedo P., Wang PM., Andreucci TH., Cenedeze MA., Teixeira VP., Reis MA., Pacheco-Silva A. and Câmara NO. (2007): Mesenchymal stem cells ameliorate tissue damages triggered by renal ischemia and reperfusion injury. Transplant. Proc., 39 (2): 421-423.

[11] Imberti B, Morigi M., Tomasoni S., Rota C., Corna D. and Longaretti L. (2007): Insulin-like growth factor-1 sustains stem cell mediated renal repair. J. Am. Soc. Nephrol., 18 (11): 2921-2928.

[12] Lange C., Togel F., Ittrich H., Clayton F., Nolte-Ernsting C. and Zander AR. (2005): Administered mesenchymal stem cells enhance recovery from ischemia/ reperfusion induced acute renal failure in rats. Kidney Int., 68:1613-1617. 
[13] Tögel F., Weiss K., Yang Y., Hu Z., Zhang P. and Westenfelder C. (2007): Vasculotropic, paracrine actions of infused mesenchymal stem cells are important to the recovery from acute kidney injury. Am. J. Physiol. Renal Physiol. 292: F1626-F1635

[14] Bi B., Schmitt R., Israilova M., Nishio H. and Cantley LG. (2007): Stromal cells protect against acute tubular injury via an endocrine effect. J. Am. Soc. Nephrol., 18 (9): 2486-2496.

[15] Lin, F., Cordes K., Li L., Hood L., Couser WG., Shankland SJ. and Igarashi P. (2003): Hematopoietic stem cells contribute to the regeneration of renal tubules after renal ischemiareperfusion injury in mice. J. Am. Soc. Nephrol., 14(5): 11881199.

[16] Imasawa T., Utsunomiya Y., Kawamura T., Zhong Y. and Nagasawa R. (2001): The potential of bone marrow-derived cells to differentiate to glomerular mesangial cells. J. Am. Soc. Nephrol. 12 (7): 1401-1409.

[17] Broekema M., Harmsen MC., Koerts JA., Petersen AH., Van Luyn MJ., Navis G. and Popa ER. (2005): Determinants of tubular bone marrow-derived cell engraftment after renal ischemia/reperfusion in rats. Kidney Int., 68: 2572-2581.

[18] Duffield JS., Park KM., Hsiao LL., Kelley VR., Scadden DT., Ichimura T. and Bonventre JV. (2005): Restoration of tubular epithelial cells during repair of the postischemic kidney occurs independently of bone marrow-derived stem cells. J. Clin. Invest., 115: 1743-1755.
[19] Thadhani R., Pascual M. and Bonventre JV. (1996): Acute renal failure. N. Engl. J. Med., 334:1448-1460.

[20] Bonventre JV. (1993): Mechanisms of ischemic acute renal failure. Kidney Int. 43 (5):1160-1178.

[21] Sutton TA. and Molitoris BA. (1998): Mechanisms of cellular injury is ischemic acute renal failure. Sem. Nephrol., 18 (5): 490-497.

[22] Kitada H., Sugitani A., Yamamoto H., Otomo N., Okabe Y., Inoue S., Nishiyama K., Morisaki T. and Tanaka M. (2002): Attenuation of renal ischemia reperfusion injury by FR167653 in dogs. Surgery, 131(6): 654-662.

[23] Scheiermann C., Colom B., Meda P., Patel NS., Voisin MB., Marrelli A. and Woodfin A. et al. (2009): Junctional adhesion molecule-C mediates leukocyte in filtration in response to ischemia reperfusion injury. Arterioscler. Thromb. Vasc. Biol., 29:1509-1515.

[24] Jang HR., Ko GJ., Wasowska BA. and Rabb H. (2009): The interaction between ischemia-reperfusion and immune responses in the kidney. J Mol. Med., 87: 859-864.

[25] Humphreys BD. and Bonventre JV. (2008): Mesenchymal stem cells in acute kidney injury. Annu. Rev. Med., 59: 311325 .

[26] Rastegar F., Shenaq D., Huang J., Zhang W., He BC., Chen L., Zuo GW., Luo Q. and Shi Q. (2010): Mesenchymal stem cells: Molecular characteristics and clinical applications. World J. Stem Cells, 2(4): 67-80. 